\title{
MOVILIZACIONES ESTUDIANTILES Y LIDERAZGO DIRECTIVO: UN ESTUDIO EXPLORATORIO EN TRES LICEOS DE LA REGIÓN METROPOLITANA
}

\author{
Javiera Peña ${ }^{1}$ y Matías Sembler ${ }^{2}$
}

\begin{abstract}
RESUMEN
El estudio de las movilizaciones estudiantiles en Chile se ha centrado en una perspectiva estructural o en el significado que le otorgan los estudiantes. Por consiguiente, el objetivo del estudio es comprender cómo se experimentan éstas desde el interior de las comunidades educativas y cómo son gestionadas por el liderazgo escolar. La investigación fue realizada mediante estudios de caso en tres escuelas de la Región Metropolitana que han experimentado movilizaciones desde el año 2006, a través de la realización de entrevistas en profundidad y análisis de contenido. Se inserta en una perspectiva que otorga relevancia a los componentes macropolíticos y micropolíticos, ahonda en los conflictos socioeducativos e indaga en las prácticas de liderazgo educativo. Los resultados permiten identificar una "normalización" de estos conflictos socioeducativos y enfatizan en sus consecuencias relacionales. Además, su gestión es identificada como una tarea central y no reconocida de los directivos, destacándose la escasez de herramientas, la preponderancia política del sostenedor y el rol intermediador de los profesores asesores del centro de estudiantes. Finalmente, se identifica un conjunto de aprendizajes y prácticas de liderazgo para una gestión más constructiva desde el liderazgo escolar.
\end{abstract}

Conceptos clave: gestión de conflictos, macropolítica escolar, micropolítica escolar, movimiento estudiantil, tomas de liceos.

\section{STUDENT MOVEMENTS AND SCHOOL LEADERSHIP: AN EXPLORATORY STUDY IN THREE HIGH SCHOOLS IN THE SANTIAGO METROPOLITAN REGION}

\begin{abstract}
The study of student mobilizations in Chile has focused on a structural perspective or on the meaning assigned to them by students. Therefore, the objective of the study is to understand how these movements are experienced within the educational communities and how they are managed by the school leadership. The research was conducted through case studies in three schools in the Santiago Metropolitan Region that have experienced mobilizations since 2006, with in-depth interviews and content analysis. The study is inserted in a perspective that emphasizes macropolitical and micropolitical components, delves into the socio-educational conflicts and explores educational leadership practices. The results identify a "normalization" of these socioeducational conflicts and emphasize their relational consequences. In addition, its management
\end{abstract}

1 Universidad Diego Portales, Santiago, Chile. Contacto: javiera.pena.fde@mail.udp.cl

2 Universidad Diego Portales, Santiago, Chile. Contacto: matias.sembler@mail.udp.cl 
is recognized as a school director's central, yet unrecognized, task. The results also highlight the lack of tools, the political preponderance of the school administrator or superintendent, and the intermediating role of the Student Center's mentor teachers. Finally, a set of learning and leadership practices are identified for a more constructive management performed by the school leadership.

Key concepts: school macropolitics and conflict management, school micropolitics, student movement, school occupation.

\section{Introducción}

Las indagaciones respecto de las movilizaciones estudiantiles en Chile generalmente han referido a un nivel estructural, destacando el papel impugnador del movimiento estudiantil respecto de la sociedad chilena en general (Donoso, 2017; Ruiz, 2016), o al sistema educativo en particular (Bellei \& Cabalin, 2013; Bellei, Cabalin \& Orellana, 2014). Este énfasis ha generado que los estudios que abordan cómo se experimentan los procesos de movilización estudiantil al interior de las comunidades educativas no hayan presentado un especial desarrollo. Es más, las investigaciones basadas en procesos de movilización en algunos establecimientos en particular (como las referentes a la cultura juvenil o a las tomas de establecimientos educacionales como repertorio de movilización) (Aguilera, 2011; Fondo de Naciones Unidas para la Infancia, Unicef, 2014), no se han centrado en comprender cómo impactan en las dinámicas que se establecen entre los diversos actores escolares.

El presente artículo plantea una aproximación a las movilizaciones estudiantiles desde las propias comunidades educativas, considerando a los múltiples actores escolares (estudiantes, directivos, docentes, auxiliares y administrativos) de tres liceos de la Región Metropolitana de Chile, que durante una década han presentado casi todos los años tomas llevadas a cabo por sus estudiantes. El foco está puesto en comprender lo que el fenómeno de las movilizaciones ha implicado para las comunidades escolares, ampliando el marco de interpretación comúnmente centrado en el simbolismo que poseen para los propios estudiantes (Aguilera, 2012; Rivera, 2013; Silva y Romero, 2013). Asimismo, se busca comprender cómo los equipos directivos afrontan las movilizaciones estudiantiles 
- y especialmente las tomas - en sus establecimientos. Con ello, no se busca presentar una postura contrapuesta a las investigaciones anteriormente reseñadas, sino ampliar el enfoque desde el cual se ha interpretado el fenómeno de las movilizaciones estudiantiles.

El artículo se inserta en una serie de esfuerzos investigativos que han buscado entender el papel del conflicto en las escuelas, comprendiendo a las instituciones educacionales como sistemas políticos, con dinámicas internas y vinculadas con el contexto social e institucional en el que se insertan (Anderson, 1996; Córdoba-Alcaide, Ortega-Ruiz y Nail-Kröyer, 2016; Davidoff \& Lazarus, 2002; Gvirtz y Beech, 2014; Ruiz, 1997; Tognetta \& Vinha, 2011). Asimismo, se reconoce la relevancia de los conflictos socioeducativos (Villalobos, Peña, Aguirre y Guerrero, 2017), buscando contribuir desde un fenómeno propio del contexto chileno — como el de los conflictos estudiantiles-, a un tema poco estudiado como ha sido el rol del liderazgo directivo en la gestión de los conflictos (Gunter, 2016).

El artículo se organiza en cinco apartados. El primero expone algunos elementos del contexto en el que se inserta el fenómeno. Posteriormente, se abordan los antecedentes conceptuales que permiten situar el análisis de las movilizaciones estudiantiles desde las comunidades escolares. El tercero presenta la metodología empleada. El cuarto apartado ofrece los resultados, agrupados en dos grandes temas:

- las características e impacto de las movilizaciones en las comunidades escolares; y

- el modo en que el liderazgo directivo en los tres liceos ha gestionado estos procesos en los últimos años.

Por último, se expone una discusión y reflexión final respecto de las principales conclusiones del estudio.

\section{Contexto}

El movimiento estudiantil de 2011 dio apertura al ciclo de movilización social de mayor envergadura experimentado tras la recuperación de la democracia en Chile (Donoso, 2017; Garretón, 
2016). Su acción contenciosa se orientó a la impugnación de políticas educacionales que consolidaban aspectos del sistema educativo ligados a las transformaciones desregularizadoras implementadas durante la dictadura militar (Bellei, 2015; Bellei et al., 2014; Fleet, 2011; Somma, 2012). Sin embargo, este auge de la política crítica del movimiento estudiantil encuentra sus antecedentes en dos movilizaciones previas, el mochilazo de 2001 y el pingüinazo de 2006, en las que los estudiantes secundarios alcanzaron preponderancia tanto en su organización como en su conducción política. Si bien desde el mochilazo ${ }^{3}$ se observa que el planteamiento de demandas al Estado constituye la principal orientación política del movimiento estudiantil (Riffo, 2013); el pingüinazo ${ }^{4}$ fue sumamente relevante para la instalación de las tomas de establecimientos como un repertorio de acción protagónico para los secundarios movilizados (Donoso, 2013).

En dicho marco, la combinación de diversas formas de movilización — tales como la realización de marchas, acciones culturales, protestas nacionales, paralización de actividades y tomas de establecimientos - han devenido en características permanentes del escenario político de la última década y, por tanto, de la realidad cotidiana de muchas escuelas (Donoso, 2013; Unicef, 2014). Junto con las demandas por cambios estructurales a las políticas educacionales y por una mayor equidad del sistema educativo en su conjunto, las organizaciones de estudiantes secundarios han desarrollado un conjunto de peticiones locales, tales como el mejoramiento de la infraestructura de los establecimientos escolares y de los beneficios estudiantiles (Unicef, 2014). Más recientemente, durante estos últimos tres años, han emergido en algunas escuelas ciertas manifestaciones caracterizadas por una mayor violencia, lo que ha llamado la atención de las autoridades de gobierno y de la

3 El mochilazo del 2001 tuvo como su principal demanda la regularización del pase escolar (Agocinos, 2013).

4 Se denominó Revolución pingüina al movimiento estudiantil secundario llevado a cabo el año 2006, el que además de demandas puntuales como la extensión del "pase escolar" (sistema tarifario rebajado de transporte público para los estudiantes) solicitaba cambios a la Ley Orgánica Constitucional de Enseñanza promulgada por Pinochet el último día de la dictadura. Se denominó pingüina porque fue liderada por los estudiantes secundarios, quienes tradicionalmente vestían entonces un uniforme escolar que asemejaba a los pingüinos en sus colores. 
opinión pública. La destrucción parcial de algunas escuelas o el uso de artefactos incendiarios, como bombas molotov en las inmediaciones han planteado importantes debates respecto de las manifestaciones y protestas de los estudiantes y de lo que ocurre al interior de las comunidades escolares.

\section{Antecedentes conceptuales}

2.1. Organizaciones escolares: entre la micro y macropolítica escolar

Desde hace algunas décadas la literatura (Anderson, 1996; Ball, 1989; Hoyle, 1986; Ruiz, 1997) ha planteado la necesidad de trascender la visión tradicional de las organizaciones escolares, centrada en su carácter racional y altamente burocrático, y ha enfatizado la perspectiva de las escuelas como sistemas políticos (González, 1998), las cuales responden tanto a sus propios procesos y dinámicas internas, como a la relación que establecen con el contexto social e institucional en el que se encuentran insertas (Anderson, 1996; Davidoff \& Lazarus, 2002). Ambas dimensiones, denominadas comúnmente como micro y macropolítica, respectivamente, son complementarias a la hora de entender la particularidad de las organizaciones escolares (Ruiz, 1997).

A fines de la década de los ochenta, diversos autores (Bacharach \& Mitchell, 1987; Ball, 1989; Blase, 1987; Hoyle, 1986) retoman la noción planteada por lannacconene (1975) acerca de las micropolíticas de la educación. Con ello, se buscaba superar el poco conocimiento que se tenía hasta entonces respecto del modo en que las escuelas funcionan cotidianamente (Ball, 1989) - lo que Hoyle denomina "el lado oscuro de la vida organizacional" (1982)—, posibilitando el reconocimiento de la existencia simultánea de diferentes (sub) culturas en las organizaciones escolares (Piot $\&$ Keltchermans, 2015). Complementariamente, este enfoque enfatizaba que los procesos de enseñanza y aprendizaje y sus resultados no respondían de forma mecánica a variables macro o de política educativa, sino que se encontraban mediados por los propios procesos organizativos de las escuelas (Blase \& Blase, 2000; Björk \& Blase, 2009; Gvirtz y Beech, 
2014; Terrén, 2004). De esta forma, la organización escolar deriva en gran medida de las propias dinámicas de cooperación y conflicto que acaecen en su interior (Blase, 2005; Ruiz, 1997) y que son resultado de la diversidad de intereses, valores, ideologías y objetivos y de la desigual distribución de poder entre sus miembros (Altrichter \& Salzberg, 2000; Ball, 1989; Blase, 2005; Contreras, 2005). Siguiendo a Blase (1991), las micropolíticas refieren al uso formal e informal del poder y a las estrategias que llevan a cabo tanto los individuos como los grupos en la persecución de sus intereses y objetivos. Desde esta perspectiva, los miembros de la escuela son identificados como actores sociales con protagonismo en la organización y no simplemente como entes sometidos a estructuras organizativas. El comportamiento micropolítico sería, entonces, una característica natural e inevitable de las organizaciones escolares (Altritcher y Salzberg, 2000; Hargreaves, 1991) y cada escuela es, en este sentido, una organización única (Ball, 1989; Guerra, 1997).

La segunda dimensión que complementa el análisis de las organizaciones escolares corresponde a la macropolítica, la cual permite establecer los vínculos existentes entre la organización escolar y los sistemas económico, político, social y legislativo (Guerra, 1997) en los que se encuentra inserta. Para Bacharach y Mundell (1993), los análisis micropolíticos requieren del análisis macropolítico, en la medida en que las escuelas no existen en el vacío, sino que responden a lo que ocurre en su entorno. El enfoque macropolítico permite visibilizar cómo las disputas llevadas a cabo principalmente a nivel del campo educativo y las lógicas de acción desplegadas por grupos de interés externos penetran en la organización (Ruiz, 1997).

De este modo, tanto los factores macro como los micropolíticos interactúan de forma constante (Blase, 1991), lo que es especialmente evidente durante períodos de reformas y cambios en la política educativa (Blase, 2005), cuando suele aumentar la intensidad de la interacción política, emergiendo y visibilizándose nuevos procesos y estructuras micropolíticas.

Ahora bien, la escuela, al ser una organización social caracterizada por el despliegue de la micropolítica y la macropolítica 
escolar, constituye un espacio donde los conflictos resultan una parte consustancial de su propia dinámica.

\subsection{Conflictos en las organizaciones escolares}

Un conflicto corresponde a una determinada forma de interacción entre distintos actores (individuales y/o colectivos), en la que al menos uno de ellos reconoce la existencia de incompatibilidades con el otro, ya sea en relación con objetivos, percepciones, formas de pensar, sentimientos y/o expectativas (Glasl, 2004; Wagner, 2005). Con el fin de analizar los conflictos que existen al interior del espacio escolar se ha propuesto el concepto de conflictos socioeducativos (Villalobos et al., 2017). Este enfatiza, en primer lugar, la estrecha vinculación entre la escuela, el campo educativo y el entorno político, cultural, social y económico en el que se encuentran los establecimientos educativos, lo que explica su componente social. En segundo lugar, destaca que los conflictos que se despliegan al interior de las escuelas tienen su particularidad propia, debido a la naturaleza organizativa del ámbito escolar, de lo que deriva su componente educativo.

Desde una perspectiva de la escuela como espacio político, el conflicto deja de ser conceptualizado como una fuerza disfuncional y negativa, propia de una visión tradicional sobre las organizaciones (Morgan, 2006), para ser entendido como natural e incluso necesario para la transformación de las estructuras educativas y el cambio hacia la mejora (Ball, 1989; Jares, 1997). El abandono de la mirada negativa de los conflictos ha permitido afianzar una perspectiva que enfatiza en la importancia y en el carácter dinamizador y creativo de los conflictos en el ámbito escolar, principalmente cuando son encauzados correctamente (Córdoba-Alcaide et al., 2016; Unline, Tschannen-Moran \& Pérez, 2003). Para ello, se requiere de liderazgos escolares que promuevan procesos de manejo y gestión (Boucher, 2013; Johnson, 2003), que posibiliten que los conflictos devengan en positivos o productivos. Entre las funciones positivas y creativas de los conflictos es posible mencionar: la promoción y emergencia de nuevas ideas, la clarificación y reevaluación de objetivos y metas, el fortalecimiento de la habilidad de los equipos para trabajar en conjunto y la liberación de tensiones al interior de las comunidades 
(Somech, 2008). Por el contrario, una gestión deficiente propiciaría la prolongación y escalamiento de los conflictos, la emergencia de actitudes y comportamientos inflexibles y hostiles y una merma en la eficacia colectiva al interior de las organizaciones (Afzalur Rahim, Magner \& Shapiro, 2000).

Las investigaciones de la gestión de conflictos, sin embargo, han tenido un papel secundario dentro del conjunto de estudios referidos al liderazgo escolar (Gunter, 2016). Durante años, diversos autores han llamado la atención respecto del poco reconocimiento otorgado por la teoría de la administración educativa a la existencia de diferencias y conflictos, y los procesos de poder e influencia en las organizaciones escolares (Blase, 1991; Hoyle, 1982; Msila, 2012; Piot \& Kelchetermans, 2015). El objetivo a continuación es exponer algunos lineamientos respecto de este tema.

\subsection{Rol del liderazgo escolar en la gestión de conflictos}

El liderazgo escolar se comprende como un proceso de influencia social que busca conducir a las escuelas hacia el logro de ciertos objetivos deseados (Bush, 2016; Leithwood, 2009). Desde un enfoque micropolítico se ha enfatizado en el rol fundamental que los directores - por su posición de autoridad- ejercen en las escuelas (Ball, 1989). Por una parte, son los llamados a mantener el control, tanto en términos organizativos (asegurando la continuidad y supervivencia) como educativos (elaboración y aplicación de una política educativa). Por otra parte, deben asegurar la adhesión e integración de sus comunidades educativas (Anderson, 1996). Asimismo, diversos estudios han dado cuenta del rol central que desempeñan en la promoción de climas positivos de convivencia escolar y confianza que conducen finalmente a mejores procesos de aprendizaje y enseñanza (Bryk \& Schneider, 2002; Bryk, Sebring, Allensworth, Luppescu \& Easton, 2010; Kutsyuruba, Walker \& Noonan, 2016).

En este marco, se ha planteado que una de las tareas del liderazgo escolar es la gestión constructiva de los conflictos al interior de las escuelas (Leithwood, 2009; Msila, 2012; Runde \& Flanagan, 2010; Unline et al., 2003). Sin embargo, el carácter normativo de 
este rol contrasta con la evidencia al respecto, que enfatiza la poca formación y herramientas con que contarían los líderes escolares en esta tarea (Anderson, 2005; Msila, 2012; Villalobos et al., 2017).

Ahora bien, esto no implica asumir que la gestión de la conflictividad escolar responda exclusivamente a las habilidades, herramientas o estilos de liderazgo ejercido por un individuo en particular como es el caso de los directores. La consecución del liderazgo escolar en esta tarea es, por una parte, resultado de un trabajo colectivo (Somech, 2008) y, a su vez, responde a las posibilidades y limitaciones planteadas por el propio contexto escolar (Anderson, 2005; Ball, 1989).

La literatura respecto de la gestión de los conflictos en el campo organizacional ha enfatizado que, para el despliegue de las estrategias de gestión constructiva de los conflictos, resulta crucial comprender la estructura de los mismos. Dana (2001) sostiene que son seis los aspectos básicos que requieren identificación y reconocimiento en el análisis de un conflicto:

- la naturaleza de la interdependencia entre las partes involucradas;

- el número de partes interesadas;

- representación de intereses;

- la(s) autoridad(es) negociadoras;

- la(s) urgencia(s) más críticas; y

- los canales de comunicación disponibles.

Asimismo, para la consecución de ambos objetivos (análisis y estrategias), el desarrollo de habilidades tanto cognitivas y emocionales como de comportamiento resulta relevante (Runde \& Flanagan, 2010). Las primeras hacen referencia al conocimiento de las actitudes y respuestas tanto propias como de otros (individuos y/o grupos) frente al conflicto. Las segundas apuntan a la comprensión de las emociones propias y de las partes involucradas, desplegando cierta capacidad de mantener un balance entre las mismas. Las últimas aluden a comportamientos que contribuyen positivamente al conflicto, tales como escuchar comprensivamente a las partes, compartir las propias opiniones y perspectivas, promover el trabajo 
conjunto en la búsqueda de soluciones y mantener las comunicaciones en marcha.

\section{Diseño metodológico}

La investigación se ha desarrollado desde un enfoque exploratorio, empleando un diseño metodológico cualitativo. El método empleado fue el estudio de caso, correspondiente a "un estudio intensivo de una unidad con el propósito de entender un conjunto mayor compuesto por unidades similares" (Gerring, 2014, p. 84). Este método permite investigar un fenómeno en su contexto, sin buscar representatividad, sino profundizando en un escenario de estudio en particular (Stake, 1995; Yin, 2004). La elección del estudio de caso se justifica, pues la investigación enfatiza en la comprensión de las movilizaciones desde dentro de las comunidades escolares.

\subsection{Técnicas de recopilación de información}

El estudio de caso se basó en información obtenida mediante entrevistas semiestructuradas individuales y grupales. La elección de la entrevista semiestructurada como técnica de investigación se fundamenta en que se buscó acceder a las diferentes experiencias de los distintos entrevistados, mediante una serie de temáticas orientadoras, lo que permite aprehender la diversidad al interior de los casos (Stake, 1995).

\subsection{Muestra y participantes}

El muestreo se realizó a dos niveles: selección de liceos y selección de actores escolares. Respecto de los primeros, estos se escogieron según características compartidas y diferenciadoras, lo que permite el despliegue de la variabilidad (Gerring, 2014). 
Tabla 1

Características compartidas y diferenciadoras de los casos estudiados ${ }^{5}$

\begin{tabular}{|c|c|}
\hline Características compartidas & Características diferenciadoras \\
\hline $\begin{array}{l}\text { - Han experimentado movilizaciones, } \\
\text { particularmente tomas, casi todos los } \\
\text { años entre } 2006 \text { y } 2017 \text {. } \\
\text { - Son liceos tradicionales y emblemáticos } \\
\text { ubicados en comunas céntricas de la } \\
\text { Región Metropolitana. } \\
\text { - Contaban con directores de cuatro años } \\
\text { de antigüedad al iniciarse el trabajo de } \\
\text { campo. }\end{array}$ & $\begin{array}{l}\text { - Uno es mixto, otro es solo de hombres y } \\
\text { el último es solo de mujeres. } \\
\text { - Las matrículas varían entre } 514 \text { y } 1.305 \\
\text { estudiantes. } \\
\text { - Dos poseen orientación científico- } \\
\text { humanista y el restante es polivalente. } \\
\text { - El año de la investigación un liceo tuvo } \\
\text { movilizaciones complejas con una toma } \\
\text { de más de un mes; otro tuvo una toma } \\
\text { de menor complejidad con duración de } \\
\text { tres días; y en el último no hubo toma, } \\
\text { pero sí otras formas de movilización, por } \\
\text { primera vez en } 11 \text { años. }\end{array}$ \\
\hline
\end{tabular}

Fuente: Elaboración propia.

La definición de los participantes se guio por dos criterios: estructural y teórico. Estructural, a fin de buscar reproducir mediante las entrevistas las distintas posiciones existentes en los liceos (Canales, 2006), para lo cual se conversó con estudiantes, directores, miembros del equipo directivo, profesores, administrativos, auxiliares y apoderados. Teórico, en tanto los hallazgos obtenidos mediante la codificación simultánea al trabajo de campo, permitió identificar los actores con quienes se debían realizar más entrevistas, pues sus perspectivas contribuían en mayor medida a densificar las categorías en construcción (Andréu Abela, García Nieto y Pérez Corbacho, 2007; Strauss \& Corbin, 2002).

5 Se ha realizado una descripción general de los liceos por principios éticos de la investigación, siendo un compromiso asumido con los equipos directivos de estos, pues corresponde a un fenómeno que ha generado diversas problemáticas y tensiones en las comunidades escolares. 
Tabla 2

Caracterización de las entrevistas realizadas

\begin{tabular}{|c|c|c|c|c|c|c|}
\hline & \multicolumn{3}{|c|}{ Caso 1} & \multicolumn{2}{|l|}{ Caso 2} & \multirow[b]{2}{*}{ 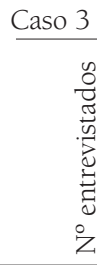 } \\
\hline & 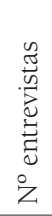 & 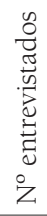 & 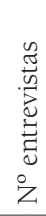 & 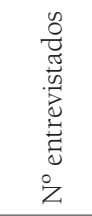 & 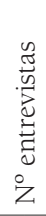 & \\
\hline Directivos & 4 & 4 & 6 & 6 & 4 & 4 \\
\hline Docentes & 6 & 5 & 4 & 4 & 4 & 5 \\
\hline Estudiantes & 8 & 16 & 3 & 5 & 4 & 7 \\
\hline No docentes & 4 & 6 & 3 & 3 & 1 & 1 \\
\hline Total por caso & 21 & 31 & 16 & 18 & 13 & 17 \\
\hline
\end{tabular}

Fuente: Elaboración propia.

\subsection{Análisis de la información}

Las entrevistas fueron transcritas y su procesamiento se realizó a través del programa de análisis de datos Nvivo 11 (2015) (Richards, 2005). Se llevó a cabo un análisis de contenido, que es un tipo particular de análisis que enfatiza en que los datos obtenidos adquieren significado según el contexto en que fueron producidos, requiriendo la construcción de categorías basadas en relaciones temáticas (Andreu Abela, 2000).

En particular, los pasos seguidos para el análisis fueron, en primer lugar, una codificación abierta, sustentada en la identificación y caracterización de categorías de análisis; para posteriormente realizar una codificación axial, orientada a relacionarlas entre sí, identificando categorías centrales y subcategorías (Strauss \& Corbin, 2002). Se realizaron sucesivas codificaciones hasta alcanzar la saturación de la información, entendida como la capacidad, por parte del investigador, de construir una perspectiva teórica consistente y convincente a partir de los datos poseídos (Martínez-Salgado, 2012). Producto de estas codificaciones se realizaron, en primer lugar, informes por cada caso, para posteriormente elaborar un análisis comparativo entre los casos, lo cual permitió identificar las tendencias comunes entre los liceos, de acuerdo con los objetivos planteados por el presente artículo. 


\subsection{Consideraciones éticas}

El diseño de la investigación fue aprobado por el Comité de Ética de la Facultad de Educación de la Universidad Diego Portales. Los participantes firmaron un formulario de consentimiento informado escrito, aceptando participar de manera voluntaria en el estudio.

\section{Resultados}

\subsection{La "normalización" de las movilizaciones estudiantiles}

La recurrencia de las movilizaciones estudiantiles, particularmente de las tomas en algunos establecimientos, permite referir a una cierta normalización de este fenómeno. Es así que, distintos actores escolares entrevistados refieren a la toma como "una tradición" (Docente, caso 1), un "hábito" (Directivo, caso 2) o "algo típico" (Estudiante, caso 1) de la vida escolar.

Yo siempre digo ¿por qué no la (la toma) planificamos en el calendario anual? ¿Por qué no ponemos "va a haber tres semanas en las cuales pueden venir a acampar, puede venir quien quiera, hacer tocatas y nos dejamos de lesear?"... Porque tú ya sabes que por $\mathrm{ABC}$ motivo va a haber algo (Docente, caso 1).

Dicha normalización se expresa en cuatro aspectos principales. Primero, en el desarrollo de un guion similar en estos procesos, el cual se concentra en el período de tres meses que antecede a las vacaciones de invierno en el país ${ }^{6}$. En dos casos, los entrevistados incluso refieren a fechas específicas, como el día de rendición de una prueba de nivel o el "día del alumno". Además, las movilizaciones siguen un patrón año a año, transitando desde un aumento de las asambleas estudiantiles hacia la realización de paros de actividades para, finalmente, generarse la toma del establecimiento:

Lo que está pasando ahora, paros internos, paros reflexivos, asambleas así de repente, CODECU ${ }^{7}$ extraordinarios, interrupción

6 Comúnmente las vacaciones de invierno transcurren durante la segunda quincena de julio.

7 Consejo de delegados de curso. 
de clases. Por ejemplo, ahora estamos en el típico escenario pretomas (Docente, caso 1).

Una segunda característica se refiere al cambio en el tipo de demandas levantadas por los estudiantes. En los primeros años de movilizaciones, las demandas externas (por ejemplo, desmunicipalización y gratuidad en la educación superior) tendían a primar — principalmente en dos de los liceos analizados- y, en torno a ellas, se solían levantar demandas internas. Sin embargo, a partir de 2012, las demandas internas (ya sea de infraestructura, curriculares o relacionales) adquirieron mayor centralidad, lo que traslada gran parte del conflicto al interior de las propias comunidades escolares.

Un tercer rasgo se refiere al cambio de la relación entre las demandas estudiantiles y la forma de movilización empleada. En los últimos cinco años ha sido común que la toma anteceda a la presentación de demandas, emergiendo como un fin en sí mismo. Para los estudiantes, esto ocurriría por la falta de espacios de articulación en contextos normales de clases. En tanto, para otros actores, referiría a una pérdida del sentido original de las tomas o a una expresión del descontento de los jóvenes con la institución escolar:

No había otra instancia en la que pudiéramos hacer las cosas, porque cuando hay paro las cosas no funcionan, cuando haces las cosas después de clases, las cosas no funcionan. Solución era la toma (Estudiante, caso 2).

En cuarto lugar, la normalización se expresa en el establecimiento de marcos regulatorios de las formas de acción de los estudiantes, tales como los "protocolos de marcha", la necesidad de contar con quorum mínimos para llevar a cabo las tomas o el acuerdo

8 Los protocolos de marcha son documentos elaborados por los establecimientos escolares con el fin de establecer ciertas normativas respecto a la autorización de sus estudiantes para asistir a marchas estudiantiles durante el horario de clases. En general, los establecimientos solicitan a los estudiantes contar con una autorización escrita y firmada por sus apoderados en que se consiente que éstos abandonen sus clases para asistir a estas manifestaciones, autorizadas previamente por la Intendencia Metropolitana. Estos protocolos comúnmente forman parte de los manuales de convivencia escolar y han sido aprobados por los distintos estamentos de la comunidad escolar (directivos, docentes, estudiantes y apoderados). 
de avisar con antelación para que directivos y docentes retiren sus pertenencias.

Hoy día se hace una, ponte tú, un CODECU y dicen toma, se vota la toma y se toman esa misma tarde el liceo. Y ahí, bueno, uno tiene que salir, y al principio las primeras tomas estaban 'fuera, fuera. Váyanse al tiro, no los queremos aquí, porque tenían como susto que los reconocieran, eso cada vez menos y entonces uno tiene tiempo para salir, yo cargo mi auto con todas las cosas más importantes, todas las llaves, todos los libros, todas las cosas (Directivo, caso 1).

Por último, las tomas se han integrado en las prácticas e imaginarios de los actores escolares. Es así que por ejemplo, los docentes han modificado sus planificaciones, concentrando evaluaciones en los dos primeros meses del año escolar. Asimismo, el ritmo de trabajo en la escuela se ha modificado, considerando contar con algunas semanas libres. Inclusive en uno de los casos, en que luego de diez años por primera vez no ocurrió una toma, los actores escolares se manifestaron más cansados por la continuidad del año.

Los profesores, yo no sé si cuando vuelven a clase estaban enojados porque los llamé a trabajar... ‘¡Tan luego!'. Yo, 'lo siento’, tengo que pensar eso. Desde el punto de vista profesional tal vez no lo debiera decir porque yo quiero a mis profes, los valoro, pero yo soy persona, a mí me pasaría lo mismo, estoy en la casa, levantándome más tarde, en invierno. Fácil (Directivo, caso 2).

\subsection{El impacto en las relaciones escolares}

En este contexto, en los tres casos se reportan efectos que han operado acumulativamente en el tiempo. Entre estos se encuentran la pérdida curricular y el impacto en los procesos de aprendizaje (con caídas importantes en la PSU y el Simce); pérdida de matrícula (estimada entre 40 a 60 estudiantes por año); pérdidas materiales y daños a la infraestructura; y mayor carga laboral para docentes y no docentes cuando finalizan estos procesos. 
Asimismo, específicamente las relaciones entre la comunidad emergen como un ámbito muy afectado. Es así como, las movilizaciones estudiantiles han generado conflictos en los liceos, agudizando también conflictos preexistentes entre los estamentos escolares o al interior de estos. Estos además, con el tránsito desde las demandas externas a las demandas internas, han tendido a incrementarse.

Si yo tuviera que hacer un gráfico de vinculación estamental en los períodos de toma, el gráfico va hacia arriba, entendiendo con la curva hacia arriba de integración interestamental cuando los petitorios son externos, y va hacia abajo, de desintegración interestamental cuando son internos (Director, caso 1).

Producto de estos conflictos han emergido emociones negativas entre los adultos, las que al no ser abordadas impactan negativamente en las relaciones entre los distintos actores escolares.

Pero ya desde más o menos el 2010 en adelante esto empezó a producir un quiebre en las confianzas, un quiebre profundo. Fue cada vez profundizándose más este quiebre hasta llegar a una etapa en la cual, y te hablo año 2016, donde literalmente uno entraba a este liceo y respiraba la tensión, a todo nivel (Directivo, caso 3).

Los conflictos emergen principalmente en tres ejes de relaciones. El primero es la relación entre docentes/no docentes y estudiantes, los cuales se deben a tres causas principales:

- las movilizaciones son asumidas por algunos adultos como un ataque personal, lo que se ve agudizado por quienes reportan situaciones violentas vivenciadas durante estos procesos;

- el mayor empoderamiento de los estudiantes genera resistencia en docentes que valoran mayormente la autoridad; y

- el cansancio por la recurrencia anual de estos procesos tensiona el ambiente. 
Claro, 'este es mi lugar, esta es mi sala, yo mando acá'. Funcionan, muchos profes, desde una visión muy autoritaria (...) Son autoritarios y por lo tanto quieren el poder, y quieren manejar, entonces, que les digan que no pueden hacer algo... es como una lógica de vieja escuela, como eso de 'cómo a ver, cabra chica, tú no me vas a decir lo que yo puedo y no puedo hacer' (Docente, caso 1).

Yo creo que en realidad más que impactar a los profesores, a nosotros como asistentes nos impactó mucho esto. ¿En qué sentido? En el sentido de que muchas veces fuimos sacadas, violentadas por los alumnos (Personal no docente, caso 3).

Un segundo eje de conflicto se produce entre los adultos, quienes suelen evidenciar diferencias de opiniones importantes respecto del fenómeno, lo que deviene en la generación de desconfianzas cruzadas. Algunos actores sospechan respecto de la instrumentalización de las movilizaciones por parte de otros grupos de adultos.

Porque a mí me tocó ver, me tocó ver, escuchar con mis propios oídos y ver con mis propios ojos a profesores diciéndoles a sus cursos que voten la toma, que llamen a toma ... Para no venir, para poner en jaque una Dirección que no le acomodaba (Docente, caso 3).

Por último, un tercer eje de conflicto se genera entre los estudiantes. Estos habrían alcanzado mayor fuerza en los últimos cuatro años, enfrentando a quienes lideran y adhieren a las movilizaciones contra quienes se oponen a estas.

Igual el año pasado hubo un incidente. Una vez un grupo se vino a tomar el colegio y entraron los estudiantes de cuarto medio a sacarlos. Entraron a la toma a sacarlos mal. Entonces, ahí tuvimos que intervenir nosotros así para que no se pusieran a pelear ni nada (Estudiante, caso 3). 
4.3. Abordando y gestionando los procesos de movilización estudiantil desde el liderazgo directivo

Al analizar cómo los equipos directivos abordan las movilizaciones estudiantiles se deben considerar las características que han adquirido estos procesos. Las movilizaciones exigen a los directivos un abordaje simultáneo de múltiples demandas y conflictos, tanto desde el interior como desde el exterior de los liceos. Desde el interior, el liderazgo directivo debe hacer frente a una micropolítica escolar altamente compleja, además de responder a una cultura organizacional estudiantil crítica de las instancias representativas. Desde el exterior, en tanto, deben enfrentar la falta de atribuciones para abordar las demandas planteadas, y la presión y el alto grado de determinación de su accionar desde la política implementada por el sostenedor.

El análisis de los casos permite identificar tres temas principales relativos al modo en que estos conflictos son abordados y gestionados. El primero de ellos, se refiere a elementos relativos al rol que asume el liderazgo directivo durante estos procesos. El segundo aborda la emergencia de una figura clave en la gestión del conflicto: los profesores asesores del Centro de Estudiantes. Por último, se proporcionan formas de acción que los propios entrevistados reconocen como aprendizajes acumulados en el tiempo.

4.3.1. El rol del liderazgo escolar en la gestión de los conflictos estudiantiles

Respecto de este punto, cinco son los principales hallazgos. El primero de ellos se refiere a la gran demanda temporal que significa la gestión de estos procesos para los directivos, quienes se ven imposibilitados de asumir otras funciones que el sistema educacional reconoce como primordiales. En particular, los directivos reconocen la dificultad para tomar un rol más pedagógico para realizar, por ejemplo, acompañamientos en el aula o participar en espacios de reflexión pedagógica. Es así como la pretensión teórica de un enfoque más pedagógico se enfrenta a la necesidad de asumir un rol más político para abordar los conflictos y las consecuencias de las movilizaciones. 
Un director aquí se le exige un carácter pedagógico... pero en estos colegios tienen que ser inminentemente político, tienes que adelantarte a las agendas para poder avanzar (Director, caso 1).

Este tipo de funciones y el rol más político de los directores tienden a ser invisibilizados por la normativa asociada al cargo. En los convenios de desempeño establecidos entre sostenedores y directores no existe un reconocimiento formal de lo que implica liderar este tipo de conflictos, y se establecen metas considerando un año común de clases y no el contexto real. Se plantea de esta forma una clara contradicción entre lo demandado al liderazgo directivo y lo que implica ejercer el liderazgo en estos liceos.

Hoy día eso tampoco es comprendido por el sistema. O sea, por ejemplo en tus convenios a ti te exigen asistencia, rendimientos, pero nadie entiende lo que significa... porque el director... está concentrado por lo menos un mes y medio (en estos procesos) y después de eso también mantener la paz... eso no lo entiende (Director, caso 2).

Un segundo hallazgo es la verticalidad con que se abordan estos conflictos. En los tres casos la gestión de las movilizaciones recae en gran medida en los directores, quienes se apoyan en una o dos personas de sus equipos, escogidos por criterios de cercanía y confianza. Esto implica que los directores suelen gestionar verticalmente estos procesos, concentrando así sus decisiones y acciones. Ellos negocian con los estudiantes, informan a la comunidad escolar y se comunican con los sostenedores. En general, el equipo directivo participa siguiendo los lineamientos del director en lugar de intervenir propositivamente en el conflicto.

Un tercer hallazgo se refiere a las escasas herramientas y conocimientos que dispondrían los directivos a la hora de gestionar estos conflictos. En primer lugar, reconocen que la poca preparación previa en temas de resolución de conflictos y la falta de conocimiento respecto de la complejidad de este fenómeno, ha redundado en que deban aprender en la práctica a lidiar con las movilizaciones. Esto conduce a que tienen que actuar sobre la base de intuiciones propias, marcadas por sus características y experiencias personales. 
Acá en este tipo de situaciones, los colegios en las tomas, yo he aprendido más que en un magíster, porque he aprendido a tener el contacto directo con la sensibilidad, el tipo que anda gestionando todo (Director, caso 2).

Un cuarto hallazgo es cómo las acciones de los directivos son más bien reactivas y se enfocan en el propio proceso de movilización, desatendiendo sus causas y consecuencias a largo plazo. Estas tienden a postergarse por la urgencia de volver al curso habitual de clases y a cumplir con lo demandado a nivel ministerial y del sostenedor.

Un último hallazgo remite a que los directores en este tipo de liceos (municipales) suelen contar con escasas atribuciones para resolver de manera efectiva los conflictos o dar respuestas a las demandas. Gran parte de las decisiones respecto de los asuntos internos, tales como temas de infraestructura o la salida de un docente, requieren del accionar de los sostenedores, lo que implica que estos asuman un mayor protagonismo en desmedro del liderazgo directivo. Esto último es más acentuado cuando, además, existen diferencias políticas y de orientación entre los sostenedores y los directores en cuanto al modo de conducir estos procesos.

En el caso de X (un curso extracurricular impartido a petición de los estudiantes), yo pido en innumerables oportunidades que me validen el pago por SEP por X, pero el sostenedor me dice que no hay recursos, yo le digo que sí hay, y él me dice que no hay y le hago dos planes alternativos de cómo poder llevar el curso $\mathrm{X}$. Nos niega todo. Y me obligó de una u otra manera, esto vía correo, mail para allá, mail para acá... a usar mis propios recursos para establecer (el curso), él nunca me dijo no hagas (el curso), inteligentemente... (Director, caso 1).

Es súper complicado para los directores... para mí el rol del director es casi nulo, estas cuestiones tienen que verlas con la corporación. Son solo cosas que puedes hablar con ellos, porque al final, son ellos los que pasan las lucas [dinero] finalmente para poder hacer todos estos cambios que se necesitan hacer ahora (Estudiante, caso 1). 
4.3.2. El profesor asesor del Centro de Estudiantes: un rol clave en la gestión del conflicto

Los entrevistados coinciden en señalar el rol clave del profesor asesor durante los procesos de movilización estudiantil. Este cargo no forma parte de los equipos directivos y cuenta solo con una asignación horaria mensual de dos horas para su ejercicio. Sin embargo, en los últimos años ha transitado de asesorar a los estudiantes en la organización de actos, celebraciones y fiestas a mediar durante las movilizaciones. Esta función no se ha reconocido formalmente, de modo que emerge como un rol difuso que depende de cómo cada docente decide ejercerlo. Por esto, desde una manera incluso más crítica que en el caso de los directores, estos profesionales no cuentan con herramientas ni formación para abordar las movilizaciones estudiantiles.

Ninguna formación. Mucha incomprensión, porque como que también yo siento que el rol del profesor dependía mucho del carácter que le pusiera tanto el profesor que iba a tomar el puesto ..., en ese sentido, uno podía ser como una contención en el sentido de frenar (a los estudiantes) en función de nuestros intereses, para otros podía ser ayudar para que cumplan sus intereses, pero no había ninguna como tipificación clara de cuál es el rol del profesor o cuál es el objetivo.

Por esto, resulta clave entender el rol que han adquirido durante estos últimos once años. Por una parte, orientan a los estudiantes, apoyándolos en la canalización de sus demandas y en la definición de sus formas de acción. Por otra parte, median entre estudiantes y otros actores escolares.

Intentando canalizar por vía relativamente pacífica, no violenta, y ojalá también armónica con lo que significa un colegio, o sea, hacer clases y todo, pero también nosotros no podíamos por ejemplo tratar a través de asesorías evitar tomar, por ejemplo, no nos correspondía, o evitar paros, sino que más bien, generar la posibilidad de que ese paro se decidiera en forma lo más transparente posible, justamente para que (el Centro de Alumnos) pudiera decir que a la decisión que llegaron fue hecha por vías 
relativamente institucionales y no a través de violencia (Docente, caso 1).

Este rol mediador los coloca en una incómoda situación intermedia entre estudiantes y autoridades, lo cual provoca un cierto aislamiento con sus pares.

4.3.3. Aprendizajes y formas de acción desplegadas en la gestión de estos conflictos

Pese a las dificultades reseñadas, se identifican prácticas de gestión que los entrevistados plantean como aprendizajes a la hora de abordar estos procesos. En particular, se enuncian siete cursos de acción y dos enfoques estratégicos de más largo plazo que, con distinto éxito, han intentado fomentar los directores en los tres liceos.

Respecto de las prácticas, destaca en primer lugar el evitar la confrontación, promoviendo el diálogo con los estudiantes movilizados. Se identifica como un aprendizaje respecto de experiencias pasadas. La actitud poco dialogante y confrontacional impulsada por directores y sostenedores principalmente en 2006 y 2011 condujo, a juicio de los entrevistados, a una agudización del conflicto con los estudiantes y a una profundización de la tensión entre adultos. Por tanto, la experiencia evidenciaría la importancia de llegar a acuerdos y ceder en algunas posturas.

Por ejemplo aquí la Dirección de estos años ha tomado el camino de la conversación. ... De llegar hasta establecer algunos acuerdos, de algunas cosas que se cumplan ¿Ya? No ir al enfrentamiento directamente con los chiquillos porque, se va como a la pérdida. Porque antes, la otra Dirección tomaba esa medida y era muy complicado (Docente, caso 3).

Una segunda práctica de gestión es el establecimiento de marcos de acción y limitaciones normativas (formales e informales) a las formas de movilización estudiantil. Destacan el establecimiento de protocolos de marcha, en que los apoderados autorizan a los estudiantes a asistir a las marchas. Pero también formas de proceder para las tomas, definiendo quórums de votación o reglas 
de comportamiento, lo que ha derivado en que disminuya su destructividad.

Ese año logramos que no se hiciera una toma a cambio de que la Dirección diera permiso para un día salir a las diez de la mañana a marchar a la Municipalidad, todo el colegio. Y fue un día súper tenso, pero resultó y fueron los chiquillos (Docente, caso 3).

Una tercera práctica refiere a la adaptación y reacción oportuna de lo que demanda la movilización estudiantil en cada momento. Los directivos sostienen que deben equilibrar la trayectoria de una década de movilizaciones con las particularidades de cada año. Por esto reconocen que las formas de acción que ayer servían, hoy ya no lo hacen, por lo que resulta clave adaptarse a la contingencia de las movilizaciones. Por ejemplo, debido a la deslegitimación de los Centros de Alumnos durante las movilizaciones, estos debieron ser capaces de vincularse con las diversas formas de organización estudiantil y sus liderazgos.

Uno también sabe perfectamente dónde están los huesos duros de roer dentro del movimiento estudiantil dentro acá del liceo. Sabe con quién hablar (...) Líderes que son naturales o creados artificialmente. Entonces, ¿qué es lo que hace uno? Uno acá tiene que tener muñeca [manejo] con y negociar, y en esos puntos claves negociar con los chiquillos, llegar a acuerdos (Directivo, caso 3).

Una quinta práctica es no involucrarse como Dirección en los rumores ni atender a acusaciones, ya sea entre estudiantes o entre adultos.

Es la Dirección la que te agudiza el conflicto, llevando la problemática de los alumnos a los adultos y con este afán de buscar responsables entre los adultos. Cuando nosotros no hemos buscado responsables, les hemos ido quitando el piso (a quienes agudizan los conflictos) (Directivo, caso 3).

Asimismo, se plantea la importancia de evitar represalias contra los estudiantes al finalizar las movilizaciones, buscando frenar el escalamiento o proliferación de conflictos posteriores. 
Hay que convencer a los profesores que no hay que tomar revancha en contra de los estudiantes. (...) es lento, pero funciona. No es fácil hacerlo (Directivo, caso 1).

Una séptima práctica refiere a evitar involucrar directamente a docentes y/o no docentes en ciertos momentos de las negociaciones con los estudiantes. Por ejemplo, en uno de los casos se destaca la importancia de evitar la presencia de docentes en un momento de alta tensión, como la entrega del liceo por parte de los estudiantes después de una toma. Respecto de una mala experiencia pasada, un directivo recuerda:

[En 2011] lo que pasa que ahí la Dirección quiso venir por los profesores y nos citó a todos a recibir el colegio, pero eso nunca tendría que haber sido. Nunca, nunca (...) o sea, yo me fui llorando de aquí. Y suponte, yo me acuerdo que íbamos caminando por el segundo piso y yo veía a mis compañeras de trabajo con lentes llorando. Porque fue una situación, si por eso te digo, fue una vulneración (tanto por el trato recibido por parte de los estudiantes como por ver directamente el estado del liceo) (Directivo, caso 2).

A estas acciones enfocadas en los procesos mismos, se suman dos estrategias signadas como relevantes por los directores, aunque han tenido mayor o menor éxito en su implementación.

La primera es el reconocimiento de la importancia de la afectividad y emocionalidad en la gestión de los conflictos. Un buen trato y mostrar preocupación por el bienestar de todos los actores escolares son señalados como elementos clave para un manejo constructivo de los conflictos y tensiones generados antes, durante y después de las movilizaciones. Especial relevancia cobra la presencialidad directiva en la cotidianeidad de la escuela.

Mucho estar en el patio. Mucho sabiendo quiénes están y también sabiendo quiénes son cada uno. Si tienes a un chico que te presenta un problema, también poder acercarte y conversar qué es lo que está pasando. (...) Y acercarte. Venir, venir, venir sin miedo. O sea, si tú les muestras miedo a los chiquillos es re difícil 
poder entrar, pero si no, van a decir 'sabe qué, yo quiero conversar con ustedes', y 'veamos, qué es lo que estás planteando, qué es lo que queremos. Conversemos' (Directivo, caso 2).

Por último, para enfrentar el impacto relacional y promover un clima positivo entre los actores escolares, los directores han buscado abrir espacios de diálogo interestamental y/o fomentar un sentido de identidad con los liceos. Ambas estrategias han tenido una efectividad variable, pues requieren recursos y tiempo del que normalmente los directores, por el ritmo y exigencias escolares, no disponen. Sin embargo, son espacios evaluados positivamente de forma transversal por los actores escolares.

Nosotros iniciamos una actividad que es casi como ya una tradición dentro del liceo. Empezamos con 'el liceo que soñamos'. (...) tenemos que ver cómo encauzar estas necesidades, estos intereses que tienen los chicos de hablar, de opinar. Y bueno, se hizo 'el liceo que soñamos'. Y, ¿por qué? Porque 'el liceo que soñamos' era tener claridad que el liceo estaba con muchos dolores, con muchos quiebres, y queríamos rescatar el sello (del liceo), qué es lo que significaba ser un (estudiante del liceo) (Directivo, caso 2).

\section{Discusión}

Las movilizaciones estudiantiles, particularmente las tomas de establecimientos, corresponden a un caso de conflicto socioeducativo (Villalobos et al., 2017), siendo un claro ejemplo de interacción entre la micropolítica y la macropolítica escolar. En primer lugar, corresponde a un conflicto con un componente marcadamente macropolítico, el cual se encuentra estrechamente ligado al desarrollo del movimiento estudiantil a nivel nacional (Björk \& Blase, 2009; Blase \& Blase, 2000; Terrén, 2004). Es así que el despliegue de un conflicto político nacional respecto de la demanda por cambios en el sistema educativo, adquiere expresiones particulares según el contexto escolar en que se desarrolla.

En segundo lugar, a dichas expresiones particulares subyace un componente micropolítico compartido, identificable en la emergencia 
de los estudiantes como un actor político, buscando materializar intereses y objetivos propios al interior de la organización escolar (Altrichter \& Salzberg, 2000; Ball, 1989; Blase, 2005; Contreras, 2005). A dichos intereses y objetivos propios se suma la toma del establecimiento como un repertorio de movilización marcadamente micropolítico, en lo referente al uso del poder formal e informal al interior de la organización escolar (Blase, 1991). Esto, pues la mencionada normalización de las tomas permite a los estudiantes ejercer un control del espacio escolar que no poseen cotidianamente. De este modo, la toma emerge como una estrategia para alcanzar cierto poder informal al interior de los liceos, en el marco de la persecución de los intereses y objetivos del actor estudiantil, puesto en disputa con los adultos que son depositarios del poder formal.

Esta reconfiguración de la micropolítica escolar con un actor estudiantil que abandona el rol tradicional más pasivo que se le ha asignado en la escuela (Neut, 2019), conlleva a que la acción de la toma del liceo devenga en conflictos tanto inter como intraestamentales. Si bien los conflictos son una parte natural de la dinámica escolar (Córdoba-Alcaide, et al., 2016; Unline, et al., 2003), las dificultades de llevar a cabo una gestión constructiva de los mismos han derivado en la agudización de la conflictividad (Rahim, Magner \& Shapiro, 2000). La normalización del fenómeno no ha significado, por tanto, su integración a la dinámica escolar de forma positiva, sino más bien se asiste a un deterioro en las relaciones y la confianza al interior de las comunidades escolares, lo que merma cualquier posibilidad de mejora escolar (Bryk \& Schneider, 2002; Kutsyuruba et al., 2016).

Los elementos que permiten explicar la ausencia de una gestión constructiva de estos conflictos responden a dos dimensiones interrelacionadas entre sí:

- el rol directivo y del liderazgo escolar; y

- el modo en el que se abordan estos conflictos en la práctica.

En relación con el rol directivo, tres son los aspectos principales a destacar. El primero de ellos es la constatación de lo ya mencionado en 
la literatura respecto de las escasas herramientas y poca formación con que cuentan quienes desempeñan cargos de liderazgo en la gestión de conflictos (Anderson, 2005; Msila, 2012, Villalobos et al., 2017). Existe entre los directivos analizados un diagnóstico común acerca de estas falencias. Resulta relevante destacar cómo entre los propios directores, después de una década de conflictividad, existe un reconocimiento en torno a la necesidad de desarrollar, en palabras de Runde y Flanagan (2010), habilidades emocionales, pero también políticas.

El segundo aspecto refiere a cómo la propia normativa asociada al rol directivo tiende a desplazar a un segundo plano la gestión de los conflictos, en aras a promover y potenciar un liderazgo escolar con mayor foco en lo pedagógico (Robinson, Lloyd \& Rowe, 2008). Esto en la práctica implica que, por una parte, los conflictos sean vistos como elementos disruptivos y negativos en la consecución de los objetivos del liderazgo escolar, alejándose de la visión que enfatiza en el potencial constructivo de los mismos (Ball, 1989; Jares, 1997; Unline et al., 2003). Por otra parte, se establece una tensión constante en los directores entre lo que realizan en la práctica debido a la gran proporción de su tiempo destinada a abordar estos conflictos-, y lo que impulsan la institucionalidad y las mismas directrices de la literatura especializada en liderazgo escolar. Esto plantea importantes desafíos a lo ya mencionado por algunos autores respecto de la necesidad de comprender, desde la política pública y la academia, al liderazgo escolar como contextualmente situado (Oplatka, 2018).

El tercer y último aspecto relativo al rol del liderazgo escolar, se refiere a la falta de atribuciones con que cuentan los directivos para gestionar y otorgar salidas a este tipo de conflictos. Si bien, por una parte, se les concede normativamente el rol de autoridad principal al interior de las escuelas (Ball, 1989), en la práctica, la preeminencia de actores como el sostenedor en el planteamiento de respuestas a demandas internas de los liceos, implica que la autoridad del director en el entramado micropolítico emerja debilitada, lo que restringe, finalmente, su capacidad de acción frente a estos conflictos. De este modo, la organización escolar (Blase, 2005; Ruiz, 1997) y los procesos micropolíticos de la escuela (Ball, 1989) se ven muy 
permeados por las orientaciones de actores más vinculados al espacio macropolítico (Bacharach \& Mundell, 1993).

El segundo elemento que permite comprender las dificultades de una gestión más constructiva de los conflictos estudiantiles y el consiguiente impacto en el entramado relacional de los liceos refiere al modo en que en la práctica se abordan estos conflictos.

Por una parte, contrariamente a lo que la literatura sugiere respecto de la gestión colectiva de estos (Somech, 2008), los directores tienden a abordar la toma y los conflictos vinculados de forma vertical e individual, concediendo ciertos grados de poder transitorio a algunos actores, tales como personas de su confianza dentro de los liceos o a los profesores asesores que emergen como figuras clave en el manejo de estos procesos. Sin embargo, la transitoriedad de esta delegación de poder genera que no se establezca una estrategia de largo plazo orientada a la gestión de conflictos.

Ligado a lo anterior, por otra parte, pareciese ser que la propia dinámica del conflicto plantea a los directores la necesidad de orientar gran parte de sus esfuerzos y escasos recursos a buscar el término de la toma, con el fin de mermar sus consecuencias curriculares (pérdida de clases) y materiales (daño de infraestructura y pérdida de material). Esto ha implicado que el foco de la acción directiva, en palabras de Dana (2001), esté puesto más en atender las urgencias más críticas que en el desarrollo de estrategias que respondan a un análisis más profundo del conflicto. Si bien, en algunos casos, los directores mencionan estrategias que buscan trascender un enfoque más coyuntural (como la medida "el liceo que soñamos"), en general, la toma es abordada como acontecimiento, soslayando el proceso más amplio en que se inserta, correspondiente a la normalización del fenómeno y a sus múltiples consecuencias acumuladas en el tiempo.

Para los directivos, finalmente, el desafío no implica solo atender los conflictos específicos que generan las tomas y su posterior gestión, sino también - y quizás en mayor medida-, abordar las relaciones al interior de las escuelas y el modo en que este fenómeno parece haber permeado la propia cultura escolar después de más de una década de conflictividad. 


\section{Conclusiones}

Considerando el escenario descrito, emergen algunos aspectos generales referidos al rol del liderazgo directivo que podrían contribuir a una gestión más constructiva de este tipo de conflictos.

Un primer aspecto relevante remite a la necesidad de una mayor coherencia entre el accionar directivo y la política implementada por el sostenedor. Esto resulta especialmente importante al tomar en consideración las limitadas atribuciones que poseen los directivos al abordar estos conflictos.

Asimismo, se plantea la necesidad de contar con una mayor distribución de funciones y responsabilidades entre el equipo directivo a la hora de abordar y gestionar las movilizaciones estudiantiles. Un primer paso sería reconocer el rol central que desempeñan los profesores asesores, esclareciendo sus funciones y mejorando sus condiciones para un adecuado desempeño. En paralelo, resulta necesario revisar la posibilidad de generar equipos de trabajo tendientes a abordar constantemente este tipo de conflictos, no actuando solo de forma reactiva.

Además, se requiere de la promoción de espacios de diálogo y reflexión en torno a estos conflictos socioeducativos de los cuales participen los distintos actores de la escuela. Esto, con miras a superar la desconfianza o las interpretaciones equívocas surgidas por el desconocimiento acerca de lo que los otros piensan o demandan. Así también, se requiere mejorar el manejo de la información con todos los actores escolares, superando la tensión caracterizada por el desconocimiento y por los rumores en un escenario de poca claridad y transparencia.

Por último, se plantea la necesidad de llevar a cabo acciones que promuevan un sentido identitario que logre aunar a las comunidades escolares, contribuyendo a que los distintos actores se sientan partícipes en la definición de objetivos compartidos, además de aminorar las tensiones generadas por las movilizaciones estudiantiles. Junto con ello, se plantean relevantes desafíos en los liceos en términos de generar espacios para la expresión política 
y cultural de los estudiantes, lo que interpela a las comunidades escolares y al sistema educativo en general.

Finalmente, para que los lineamientos previamente señalados puedan desarrollarse con mayor efectividad en los liceos que han experimentado un proceso de normalización de las movilizaciones estudiantiles, resulta necesario ahondar en el fenómeno de las tomas de establecimientos, abordando las motivaciones y los significados que este repertorio de movilización ha adquirido para los estudiantes. Este tema excede los objetivos del presente artículo, sin embargo, constituye un aspecto central a la hora de identificar posibles soluciones a este tipo de conflictos, resultando primordial que sea abordado en futuras investigaciones.

\section{Referencias}

Aguilera, O. (2011). Acontecimiento y acción colectiva juvenil. El antes, durante y después de la rebelión de los estudiantes secundarios chilenos en el 2006. Propuesta Educativa, 35, 11-26.

Aguilera, O. (2012). Repertorios y ciclos de movilización juvenil en Chile (2000-2012). Utopía y Praxis Latinoamericana, 17(57), 101-108.

Altrichter H. \& Salzberg, S. (2000). Some elements of a micro-political theory of school development. En H. Altrichter \& J. Elliot (Eds.), Images of educational change. (pp. 99-110). Oxford: Marston Lindsay Ross International.

Anderson G. L. (1996). The cultural politics of schools: Implications for leadership. En K. Leithwood, J. Chapman, D. Corson, P. Hallinger, \& A. Hart (Eds.), International handbook of educational leadership and administration. Kluwer International Handbooks of Education, vol 1. (pp. 947-966). Dordrecht: Springer.

Anderson, M. (2005). Conflict management and the prospective principal. Academic Exchange Quarterly Summer, 9(2), 146-151.

Andreú Abela, J. (2000). Las técnicas de análisis de contenido: una revisión actualizada. Fundación Centro Estudios Andaluces, Universidad de Granada, 10(2), 1-34. Recuperado de http://public. centrodeestudiosandaluces.es/pdfs/S200103.pdf

Andréu Abela, J., García Nieto, A., y Pérez Corbacho, A. M. (2007). Cuadernos metodológicos 40. Evolución de la teoría fundamentada como técnica de análisis cualitativo. Madrid: CIS. 
Bacharach, S. B. \& Mitchell, S. M. (1987). The generation of practical theory: Schools as political organizations. En J. W. Lorsch (Ed.), Handbook of organizational behavior (pp. 405-418). Englewood Cliffs: Prentice Hall.

Bacharach, S. B. \& Mundell, B. L. (1993). Organizational politics in schools: Micro, macro, and logics of action. Educational Administration Quarterly, 29(4), 423-452. https://doi.org/10.1177/0013161x93029004003

Ball, S. (1989). La micropolitica de la escuela. Barcelona: Ediciones Paidós Ibérica.

Bellei, C. (2015). El gran experimento: mercado y privatización de la educación chilena. Santiago de Chile: LOM Ediciones.

Bellei, C. \& Cabalin, C. (2013). Chilean student movements: Sustained struggle to transform a market-oriented educational system. Current Issues in Comparative Education, 15(2), 108-123.

Bellei, C., Cabalin, C., \& Orellana, V. (2014). The 2011 Chilean student movement against neoliberal educational policies. Studies in Higher Education, 39(3), 426-440. https://doi.org/10.1080/03075079.2014 .896179

Blase, J. (1987). Political interaction among teachers: Sociocultural contexts in the schools. Urban Education, 22(3), 286-309. https://doi. org/10.1177/004208598702200302

Blase, J. (1991). The politics of life in schools: Power, conflict, and cooperation. Newbury Park: Sage Publications.

Blase J. (2005). The micro politics of educational change. En A. Hargreaves (Ed.), Extending educational change (pp. 264-277). Dordrecht: Springer.

Blase, J. \& Blase, J. (2000). The micro politics of instructional supervision: A call for research. Educational Administration Quarterly, 38(1), 6-44. https://doi.org/10.1177/0013161X02381002

Björk, L. G. \& Blase, J. (2009). The micro politics of school district decentralization. Educational Assessment, Evaluation and Accountability, 21(3), 195-208. https://doi.org/10.1007/s11092-009-9078-y

Boucher, M. (2013). The relationship of principal conflict management style and school climate (Tesis de doctorado), University of South Caroline, Columbia, SC, Estados Unidos.

Bryk, A. \& Schneider, B. (2002). Trust in schools: A core resource for improvement. New York: Russell Sage Foundation.

Bryk, A., Sebring, P., Allensworth, E, Luppescu, S., \& Easton, J. (2010) Organizing schools for improvement: Lessons from Chicago. Chicago: The University of Chicago Press. 
Bush, T. (2016). Mejora escolar y modelos de liderazgo: hacia la comprensión de un liderazgo efectivo. En J. Weinstein (Ed.), Liderazgo educativo en la escuela. Nueve miradas (pp. 19-44). Santiago de Chile: Ediciones UDP.

Canales, M. (2006). Presentación. En M. Canales (Ed.), Metodologías de la investigación social. Introducción a los oficios (pp. 11-31). Santiago de Chile: LOM Ediciones.

Contreras, B. (2005). Micropolítica escolar: estilo de liderazgo de una directora y participación de docentes y alumnos en la gestión escolar. Estudio de caso en un centro educativo de nivel secundario de Lima Norte (Tesis de maestría) Pontificia Universidad Católica del Perú, Lima, Perú.

Córdoba-Alcaide, F., Ortega-Ruiz, R., y Nail-Kröyer, O. (2016). Gestión de la convivencia y afrontamiento de la conflictividad escolar y el bullying. Santiago de Chile: RIL Editores.

Dana, D. (2001). Conflict resolution: Mediation tools for everyday worklife. New York: McGraw-Hill Professional.

Davidoff, S. \& Lazarus, S. (2002). The learning school: An organization development approach. Lansdowne: Juta.

Donoso, S. (2013). Dynamics of change in Chile: Explaining the emergence of the 2006 Pingüino Movement. Journal of Latin American Studies, 45(1), 1-29. https://doi.org/10.1017/s0022216x12001228

Donoso, S. (2017). "Outsider" and "insider" strategies: Chile's student movement, 1990-2014. En S. Donoso \& M. von Bülow (Ed.), Social movements in Chile. organization, trajectories, and political consequences (pp. 65-97). Brasilia: Palgrave Macmillan.

Fleet, N. (2011). Movimiento estudiantil y transformaciones sociales en Chile: una perspectiva sociológica. Polis. Revista Latinoamericana, 10(30), 99116. https://doi.org/10.4067/s0718-65682011000300005

Fondo de las Naciones Unidas para la Infancia, Unicef. (2014). La voz del movimiento Estudiantil 2011. Educación Pública, Gratuita y de Calidad. Algunas lecciones para el sistema educativo. Santiago de Chile: Edición Miguel Lafferte y Carolina Silva E.I.R.L.

Garretón, M. A. (2016). La gran ruptura. Institucionalidad politica y actores sociales en el Chile del siglo XXI. Santiago de Chile: LOM Ediciones.

Gerring, J. (2014). ¿Qué es un estudio de caso y para qué sirve? En R. Castiglioni y C. Fuentes (Eds.), Politica comparada sobre América Latina: teorías, tópicos y método. Santiago de Chile: Editorial UDP.

Glasl, F. (2004). Konfliktmanagement. Ein Handbuch für Führungskräfte, Beraterinnen und Berater. Aktualisierte Auflage. Berlin: Hauptverlag. 
González, M. (1998). La micropolítica de las organizaciones escolares. Revista de Educación, 316, 215-239.

Guerra, M. (1997). La luz del prisma: para comprender las organizaciones educativas. Málaga: Ediciones Aljibe.

Gunter, H. (2016). An intellectual history of school leadership practice and research. London: Bloomsbury Publishing.

Gvirtz, S. y Beech, J. (2014). Educación y cohesión social en América Latina: una mirada desde la micropolítica escolar. Education Policy Analysis Archives/Archivos Analíticos de Políticas Educativas, 22, 1-24. http://doi. org/10.14507/epaa.v22n44.2014

Hargreaves, A. (1991). Contrived collegiality: The micro politics of teacher collaboration. En J. Blase (Ed.), The politics of life in schools: Power, conflict, and cooperation (pp. 46-72). Newbury Park: Sage Publications.

Hoyle, E. (1982). Micro-politics of educational organizations. Educational Management and Administration, 10(2), 87-98. https://doi. org/10.1177/174114328201000202

Hoyle, E. (1986). The politics of school management. London: Hodder and Stoughton.

Iannaccone, L. (1975). Educations policy systems: A study guide for educational administrators. Fort Lauderdale: Nova University.

Jares, X. (1997). El lugar del conflicto en la organización escolar. Revista Iberoamericana de Educación, 15(1), 53-74.

Johnson, P. (2003). Conflict and the school leader: Expert or novice. Journal of Research for Educational Leaders, 1(3), 28-45.

Kutsyuruba, B., Walker, K., \& Noonan, B. (2016). The trust imperative in the school principalship: The Canadian perspective. Leadership and Policy in Schools, 15(3), 343-372. https://doi.org/10.1080/15700763 .2016.1164866.

Leithwood, K. (2009). ¿Cómo liderar nuestras escuelas? Aportes desde la investigación. Santiago de Chile: Fundación Chile y Fundación CAP.

Martínez-Salgado, C. (2012). El muestreo en investigación cualitativa: principios básicos y algunas controversias. Ciência \& Saúde Coletiva, 17(3), 613-619. http://doi.org/10.1590/S1413-81232012000300006

Morgan, G. (2006). Images of organization. Beverly Hills: Sage Publications

Msila, V. (2012). Conflict management and school leadership. Journal of Communication, 3(1), 25-34. 
Neut, P. (2019). Contra la escuela. Autoridad, democratización y violencias en el escenario educativo chileno. Santiago de Chile: LOM Ediciones.

Nvivo (2015) Nvivo (11) [Software]. Recuperado de http://www. qsrinternational.com/nvivo/nvivo-products/nvivo-11-for-windows/ nvivo-pro

Oplatka, I. (2018). Educational administration and the relational approach: Can we suffice contextual-based knowledge production? En S. Eacott (Ed.), Beyond leadership (pp. 199-210). Singapore: Springer. https:// doi.org/10.1007/978-981-10-6568-2_12

Piot, L. \& Kelchtermans, G. (2016). The micro politics of distributed leadership: Four case studies of school federations. Educational Management Administration E Leadership, 44(4), 632-649. https://doi. org/10.1177/1741143214559224

Rahim, A., Magner, N., \& Shapiro, D. (2000). Do justice perceptions influence styles of handling conflict with supervisors? What justice perceptions, precisely? International Journal of Conflict Management, 11(1), 9-31. https://doi.org/10.1108/eb022833

Richards, L. (2005). Handling qualitative data: A practical guide. London, Thousand Oaks, New Delli, Singapore: Sage Publications.

Riffo, M. (2013). Movimiento estudiantil, sistema educativo y crisis política actual en Chile. Polis, 12(36), 223-240. https://doi.org/10.4067/s071865682013000300010

Rivera, S. (2013). El movimiento estudiantil chileno desde una perspectiva participativa. Revista Búsquedas Políticas, 2(1), 49-56.

Robinson, V. M., Lloyd, C. A., \& Rowe, K. J. (2008). The impact of leadership on student outcomes: An analysis of the differential effects of leadership types. Educational Administration Quarterly, 44(5), 635-674. https://doi. org/10.4135/9781473915152.n6

Ruiz, T. (1997). Teoría y práctica de la micropolítica en las organizaciones escolares. Revista Iberoamericana de Educación, 15, 13-52.

Ruiz, C. (2016). Crisis política en Chile: neoliberalismo, cambios sociales y democracia. En M. A. Garretón (Coord.), La gran ruptura (pp. 83-108). Santiago de Chile: LOM Ediciones.

Runde, C. E. \& Flanagan, T. A. (2010). Developing your conflict competence: A hands-on guide for leaders, managers, facilitators, and teams. San Francisco: Jossey-Bass.

Silva, C. y Romero, J. (2013). ¿Qué es un movimiento social fuerte? Conceptualizaciones de la noción de fortaleza y aplicación al caso del 
movimiento estudiantil 2002-2011 en Chile. Última Década, 21(38), 91-108. http://dx.doi.org/10.4067/S0718-22362013000100005

Strauss, A. \& Corbin, J. (2002). Bases de la investigación cualitativa. Técnicas y procedimientos para desarrollar la teoría fundamentada. Medellín: Editorial Universidad de Antioquia.

Somech, A. (2008). Managing conflict in school teams: The impact of task and goal interdependence on conflict management and team effectiveness. Educational administration quarterly, 44(3), 359-390. https://doi. org/10.1177/0013161x08318957

Somma, N. (2012). The Chilean student movement of 2011-2012: Challenging the marketization of education. Interface, 4(2), 296-309.

Stake, R. (1995). The art of case study research. Thousand Oaks: Sage Publications.

Terrén, E. (2004). Las organizaciones educativas como sistemas de comunicación. Un enfoque micropolítico. Revista Iberoamericana de Educación, 36, 189-214.

Tognetta, L. \& Vinha, T. (Orgs.) (2011). Conflitos na instituição educativa: Perigo ou oportunidade. Contribuições da psicologia. Campinas: Mercado de Letras.

Unline, C., Tschannen-Moran, M., \& Perez, L. (2003). Constructive conflict: How controversy can contribute to school improvement. Teachers College Record, 105(5), 782-815. https://doi.org/10.1111/14679620.00268

Villalobos, C., Peña, J., Aguirre, E., y Guerrero, M. (2017). Liderazgo escolar y conflictos socioeducativos. Un estudio exploratorio en liceos públicos chilenos. Calidad en la Educación, 47, 81-111.

Wagner, U. (2005). Konfliktforschung und Friedenssicherung. En D. Frey \& C. Hoyos (Hrsg.), Psychologie in Gesellschaft, Kultur und Umwelt. Handbuch (pp. 55-60). Weinheim: Beltz, PVU.

Yin, R. (1994). Case study research: Design and method. Thousand Oaks: Sage Publications. 